\title{
An alternative formula for approximate extended Born inversion
}

\author{
Jie Hou ${ }^{1}$ and William W. Symes ${ }^{1}$
}

\begin{abstract}
Various modifications of reverse time migration (RTM) provide asymptotic inverses to the subsurface offset extended Born modeling operator for constant-density acoustics. These approximate inverses have the same quality (asymptotic accuracy) as do generalized Radon transform pseudoinverses, but they can be computed without any ray tracing whatsoever. We have developed an approximate inverse of this type whose additional computational cost, above that of subsurface offset extended RTM, is negligible.
\end{abstract}

\section{INTRODUCTION}

Reverse time migration (RTM) with space-shift imaging condition (Sava and Fomel, 2003) is the transpose or adjoint of the spaceshift (or subsurface offset) extended Born modeling operator of constant-density acoustics (Stolk et al., 2009). Remarkably, preand postmultiplication with relatively low-cost auxiliary operators convert the RTM operator from an adjoint into an asymptotic inverse operator. This observation is implicit in a few works on "true amplitude migration" (Zhang et al., 2007; Xu et al., 2011), and it was made explicit by ten Kroode $(2012,2014)$ and Hou and Symes (2015b). In one of its forms, the approximate inverse is

$$
\bar{F}^{\dagger}=W_{\text {model }}^{-1} \bar{F}^{*} W_{\text {data }},
$$

in which $\bar{F}$ is the subsurface offset extended Born modeling operator (the dependence on the background model is suppressed) and $\bar{F}^{*}$ is its adjoint (a form of RTM). The functions $W_{\text {model }}$ and $W_{\text {data }}$ are the model- and data-domain weight operators, defined by

$$
\begin{aligned}
W_{\text {model }}^{-1} & =4 v^{5}\left(D_{x}^{2}+D_{z}^{2}\right)^{1 / 2}\left(D_{h}^{2}+D_{z}^{2}\right)^{1 / 2} P ; \\
W_{\text {data }} & =I_{t}^{4} D_{z_{s}} D_{z_{r}} .
\end{aligned}
$$

Here, $v$ is the background or migration velocity $(v(x, z)$ in $2 \mathrm{D}$ and $v(x, y, z)$ in 3D); $D_{x}, D_{h}$, and $D_{z}$ are the $x$-, $h$-, and $z$-direction derivatives, respectively (where $h$ is the subsurface offset), applied in the image domain; $I_{t}$ is the indefinite time integral; $D_{z_{s}}$ and $D_{z_{r}}$ are the source and receiver depth derivatives, respectively, applied in the data domain; $P(\ldots)$ (the reciprocal is used in Hou and Symes, 2015b) is a so-called pseudodifferential operator ( $\Psi D O)$ of order zero, an oscillatory integral defined by its symbol or amplitude, also denoted as $P$ (see equation 11 below); and $\bar{F}^{\dagger}$ is an asymptotic inverse in the sense that $\bar{F}^{\dagger} \bar{F}-I$ suppresses highfrequency components, i.e., in the same sense as the generalized Radon transform (GRT) or Kirchhoff inverses (Beylkin, 1985; Bleistein, 1987; Operto et al., 2000). Of course, GRT inverses require extensive computation of traveltime and amplitude tables. The operator $\bar{F}^{\dagger}$ requires no ray-related computations at all.

Note that the operator $W_{\text {model }}$ defined by Hou and Symes (2015b) is written here as $W_{\text {model }}^{-1}$, so that $\bar{F}^{\dagger}$ is expressed as the adjoint of $\bar{F}$ with respect to weighted norms. This observation leads to a dramatic acceleration of iterative extended least-squares migration (Hou and Symes, 2016a).

In this paper, we derive and illustrate an alternative approximate inverse

$$
\bar{F}^{\ddagger}=-8 v^{4} D_{z} Q \bar{F}^{*} D_{t} W_{\text {data }},
$$

in which $Q$ is a $\Psi \mathrm{DO}$ with properties similar to those of $P$. We show that $\bar{F}^{\ddagger}$ is an asymptotic inverse in the same sense as is $\bar{F}^{\dagger}$, and we illustrate its equivalent accuracy with a nontrivial numerical example. However, note that the expression 3 for $\bar{F}^{\ddagger}$ does not take the form of an adjoint of $\bar{F}$ with respect to weighted norms.

The steps necessary to evaluate the right sides of equations 1 and 3 are as follows:

1) Apply the weight operator $W_{\text {data }}$ to the data. Our previous papers (Hou and Symes, 2014, 2015b) discuss implementation of this step. For example, for shallow tow-depth streamer data (free surface, small $z_{s}, z_{r}$ relative to the shortest wavelength to be imaged), the vertical source and receiver derivatives are present in the recorded traces already, as a side effect of ghosting.

Manuscript received by the Editor 21 June 2016; revised manuscript received 1 August 2016; published online 26 October 2016.
${ }^{1}$ Rice University, Department of Earth Science, Houston, Texas, USA. E-mail: jie.hou@ rice.edu; symes@caam.rice.edu.

(C) 2017 Society of Exploration Geophysicists. All rights reserved. 
If the data are free of ghost reflections, shift the vertical derivatives to the migration using a dipole Green's function in RTM.

2) For equation 3: Apply the time derivative, or include one less time integration in the definition of $W_{\text {data }}$.

3) Apply the extended RTM operator $\bar{F}^{*}$, formulated with absorbing boundary if the source and receiver vertical derivatives are already applied to the data, otherwise formulated with the vertical dipole source and receiver.

4)

a) For equation 1: Apply square roots of Laplacians in $x, z$ and $h, z$ (2D) or $x, y, z$ and $h_{x}, h_{y}, z$ (3D). This step requires partial Fourier transforms of $3 \mathrm{D}$ data (for $2 \mathrm{D}$ inversion) or 5D data (for 3D inversion).

b) For equation 3: Apply the z-derivative.

5) Apply the operators $P$ (equation 1) or $Q$ (equation 3), or appropriate approximations.

6) Scale by appropriate power of $v$ and numerical factors.

For kinematically accurate $v$, the output of $\bar{F}^{*}$ focuses near $h=0$, and an appropriate approximation for $P$ or $Q$ is the identity operator, i.e., a "no-op." We will say a few words about the general case in the "Discussion" section.

Comparison of steps $4 \mathrm{a}$ (for equation 1 ) and 2 and $4 \mathrm{~b}$ (for equation 3 ) suggests that implementation of equation 3 is likely to be less computationally demanding than implementation of equation 1 because no spatial transforms are involved in the former implementation. At least for kinematically accurate migration velocity, with $Q$ neglected, equation 3 defines a least expensive RTM-based approximation inverse, as computationally feasible as subsurface-offset extended RTM itself.

In the next section, we will outline the theoretical derivation of equations 1 and 3, limiting ourselves to 2D for simplicity. In the following section, we present an example based on the Marmousi model (Bourgeois et al., 1991). The "Discussion" section points out some open questions about the constructions described here.

\section{THEORY}

In this section, we sketch an approach for construction of approximate inverse operators, reserving mathematical details for the appendices. We make extensive use of results from Hou and Symes (2015b). To avoid confusion, we prefix all citations of equations from that paper by "HS-." We also use the notation of that paper, in particular $\phi\left(x_{r}, x_{s}, x, z, h\right)=T\left(x_{s}, z_{s}, x-h, z\right)+T\left(x_{r}, z_{r}, x+h, z\right)$ for the offset reflection time as a function of the source location $\left(x_{s}, z_{s}\right)$, receiver location $\left(x_{r}, z_{r}\right)$, midpoint $(x, z)$, and subsurface offset $h$. Source and receiver depths are idealized as being the same for all traces, so we drop $z_{s}, z_{r}$ from the argument list of $\phi$. We assume for simplicity that $\partial \phi / \partial z>0$ in the domain of interest, i.e., that the reflection time is increasing with depth throughout the region of nonzero reflectivity. This assumption is consistent with the use of horizontal subsurface offset.

The key computation is the asymptotic approximation of a GRT of the special form

$$
\begin{aligned}
M_{a} u(x, z, h) & =\int d x_{r} d x_{s} d x^{\prime} d z^{\prime} d h^{\prime} a\left(x_{r}, x_{s}, x, z, h, x^{\prime}, z^{\prime}, h^{\prime}\right) \\
& \times \delta\left(\phi\left(x_{r}, x_{s}, x, z, h\right)-\phi\left(x_{r}, x_{s}, x^{\prime}, z^{\prime}, h^{\prime}\right)\right) u\left(x^{\prime}, z^{\prime}, h^{\prime}\right),
\end{aligned}
$$

defined by the phase $\phi$ and an amplitude function $a$. Appendix A shows that

$$
\begin{aligned}
& M_{a} u(x, z, h) \\
& \quad \approx-\frac{1}{4 \pi} \int d k_{x} d k_{z} d k_{h} e^{i\left(x k_{x}+z k_{z}+h k_{h}\right)} \frac{\hat{u}\left(k_{x}, k_{z}, k_{h}\right)}{Q\left(x, z, h, k_{x} / k_{z}, k_{h} / k_{z}\right)} \\
& \quad \times\left[\frac{v^{2}}{k_{z}^{2}} \frac{\partial \phi}{\partial z}\left(\frac{\partial \alpha_{s}}{\partial x_{s}} \frac{\partial \alpha_{r}}{\partial x_{r}}\right)^{-1} a\right]\left(x_{r}\left(x, z, h, k_{x} / k_{z}, k_{h} / k_{z}\right)\right. \\
& \left.\quad x_{s}\left(x, z, h, k_{x} / k_{z}, k_{h} / k_{z}\right), x, z, h\right) .
\end{aligned}
$$

In this representation, $\hat{u}$ denotes the Fourier transform of $u$ and $Q\left(x, z, h, k_{x} / k_{z}, k_{h} / k_{z}\right)$ is a symbol of order zero, i.e., the amplitude (nonoscillatory) factor in the standard representation of a $\Psi D O$. Properties of symbols are described in many standard texts, e.g., Taylor (1981). The value $Q$ is defined explicitly in Appendix A and satisfies

$$
\begin{aligned}
& Q\left(x, z, h, k_{x} / k_{z}, k_{h} / k_{z}\right) \neq 0 \text { if } k_{z} \neq 0, \\
& Q\left(x, z, 0, k_{x} / k_{z}, k_{h} / k_{z}\right) \equiv 1 .
\end{aligned}
$$

The term in brackets is a function of the source and receiver locations $x_{r}, x_{s}$ that are in turn functions of midpoint and offset coordinates and wavenumbers. This functional relationship depends on determining the takeoff slowness vectors $\nabla_{x, z} T\left(x_{r, s}, z_{r, s}, x \pm h, z\right)$ of rays connecting sources and receivers to scattering points, from the stationary phase conditions that lead to the asymptotic formula 5. The details are given in ten Kroode (2012) and Appendix A of Hou and Symes (2015b) and are also sketched in Appendix A.

A convenient starting point for the derivation of formulas 2 and 3 is equation HS-20, expressing the extended Born operator applied to an extended perturbation $\delta \bar{v}(x, z, h)$ of the (nonextended) velocity model $v(x, z)$, modified by time integrations and vertical source and receiver derivatives, and approximated by ray theory Green's functions. As in Hou and Symes (2015b), we adopt the notation convention that $\theta_{r}=\theta\left(x_{r}, z_{r}, x+h, z\right)$ for the surface takeoff angle of the ray from $(x+h, z)$ to the receiver at $\left(x_{r}, z_{r}\right)$, and similarly for $\theta_{s}$ and other functions of source and receiver locations and scattering points, with primes for functions of primed variables. The result of equation HS-20 is (we reproduce it here)

$$
\begin{aligned}
& I_{t} D_{z_{s}} I_{t} D_{z_{r}} I_{t} \bar{F} \delta \bar{v}\left(x_{r}, x_{s}, t\right) \\
& \quad \approx \int d x d z d h a_{s} a_{r} \frac{\cos \theta_{r}}{v_{r}} \frac{\cos \theta_{s}}{v_{s}} \delta(t-\phi) \frac{2 \pi \delta \bar{v}}{v^{3}}(x, z, h) .
\end{aligned}
$$

Set

$$
a^{\dagger}=-a_{s} a_{r} a_{s}^{\prime} a_{r}^{\prime} \frac{\cos \theta_{r}}{v_{r}} \frac{\cos \theta_{s}}{v_{s}} \frac{4 \pi^{2}}{v^{3} v^{\prime 3}} .
$$

Equation 7 implies that

$$
\left(I_{t} \bar{F}\right)^{*}\left(I_{t} D_{z_{s}} I_{t} D_{z_{r}} I_{t} \bar{F}\right) \approx M_{a^{\dagger}} .
$$


The fact $I_{t}^{*}=-I_{t}$ and application of approximation 5 turns equation 9 into equation HS-21 (the derivation details can be found in Appendix A of Hou and Symes, 2015b):

$$
\begin{aligned}
M_{a^{\dagger}} \approx & -\frac{1}{32 v(x, z)^{5} \pi^{3}} \int d k_{x} d k_{z} d k_{h} e^{i\left(k_{x} x+k_{z} z+k_{h} h\right)} \\
& \times \frac{\widehat{\delta \bar{v}}\left(k_{x}, k_{z}, k_{h}\right)}{k_{x z} k_{h z} P\left(x, z, h, k_{x}, k_{z}, k_{h}\right)} .
\end{aligned}
$$

Here, $k_{x z}=\sqrt{k_{x}^{2}+k_{z}^{2}}$ and $k_{h z}=\sqrt{k_{h}^{2}+k_{z}^{2}}$ are the symbols of the operator square roots in the definition of $W_{\text {model }}^{-1}$. Now invoke the multiplicative property of $\Psi D O s:$ To the leading order in frequency, the symbol of the product (composition) of YDOs is the (numerical) product of the symbols. That is, $1 / k_{x z} k_{h z}$ is canceled by applying $W_{\text {model }}^{-1}$, and $1 / P$ by applying the $\Psi$ DO

$$
\begin{aligned}
(P u)(x, z, h)= & \frac{1}{8 \pi^{3}} \int d k_{x} d k_{z} d k_{h} e^{i\left(k_{x} x+k_{z} z+k_{h} h\right)} \\
& \times P\left(x, z, h, k_{x}, k_{z}, k_{h}\right) \hat{u}\left(k_{x}, k_{z}, k_{h}\right) .
\end{aligned}
$$

Because $W_{\text {data }}$ is already implicit in the left side of equation 9, the approximate inverse 1 is established.

To derive the approximate inverse 3 , note that

$$
\frac{\partial}{\partial t} \delta(t-\phi)=-\left(\frac{\partial \phi}{\partial z}\right)^{-1} D_{z} \delta(t-\phi)
$$

Differentiate both sides of equation 7 with respect to $t$, use equation A-1, and integrate by parts over $z$ to get

$$
\begin{aligned}
& D_{t} I_{t} D_{z_{s}} I_{t} D_{z_{r}} I_{t} \bar{F} \delta \bar{v}\left(x_{r}, x_{s}, t\right) \\
& \quad \approx-\int d x d z d h a_{s} a_{r} \frac{\cos \theta_{r}}{v_{r}} \frac{\cos \theta_{s}}{v_{s}}\left(\frac{\partial \phi}{\partial z}\right)^{-1} \\
& \quad \times \delta(t-\phi) \frac{2 \pi D_{z} \delta \bar{v}}{v^{3}}(x, z, h) .
\end{aligned}
$$

Accordingly,

$$
\left(I_{t} \bar{F}\right)^{*}\left(D_{t} I_{t} D_{z_{s}} I_{t} D_{z_{r}} I_{t} \bar{F}\right) \approx M_{a^{\ddagger}}
$$

with

$$
a^{\ddagger}=a_{s} a_{r} a_{s}^{\prime} a_{r}^{\prime} \frac{\cos \theta_{r}}{v_{r}} \frac{\cos \theta_{s}}{v_{s}}\left(\frac{\partial \phi^{\prime}}{\partial z}\right)^{-1} \frac{4 \pi^{2}}{v^{3} v^{\prime 3}} D_{z} .
$$

Another application of the approximation 5, detailed in Appendix B, leads to

$$
\begin{aligned}
& 8 v(x, z)^{4}\left(I_{t} \bar{F}\right)^{*}\left(D_{t} I_{t} D_{z_{s}} I_{t} D_{z_{r}} I_{t} \bar{F}\right) \delta \bar{v}(x, z, h) \\
& \simeq \frac{1}{8 \pi^{3}} \int d k_{x} d k_{z} d k_{h} e^{i\left(x k_{x}+z k_{z}+h k_{h}\right)} \frac{\widehat{\delta \bar{v}}\left(k_{x}, k_{z}, k_{h}\right)}{i k_{z} Q\left(x, z, h, k_{x} / k_{z}, k_{h} / k_{z}\right)} .
\end{aligned}
$$

Using the fact $I_{t}^{*}=-I_{t}$ and the multiplicative property of $\Psi D O s$ as before to move $D_{z}$ (symbol $i k_{z}$ ) and $Q$ to the left side leads directly to equation 3 .

\section{NUMERICAL EXAMPLE}

In this section, we use the synthetic Marmousi model (Bourgeois et al., 1991) to compare the accuracy of the two approximate inverse formulas. We use a kinematically accurate velocity; in fact, we gen-
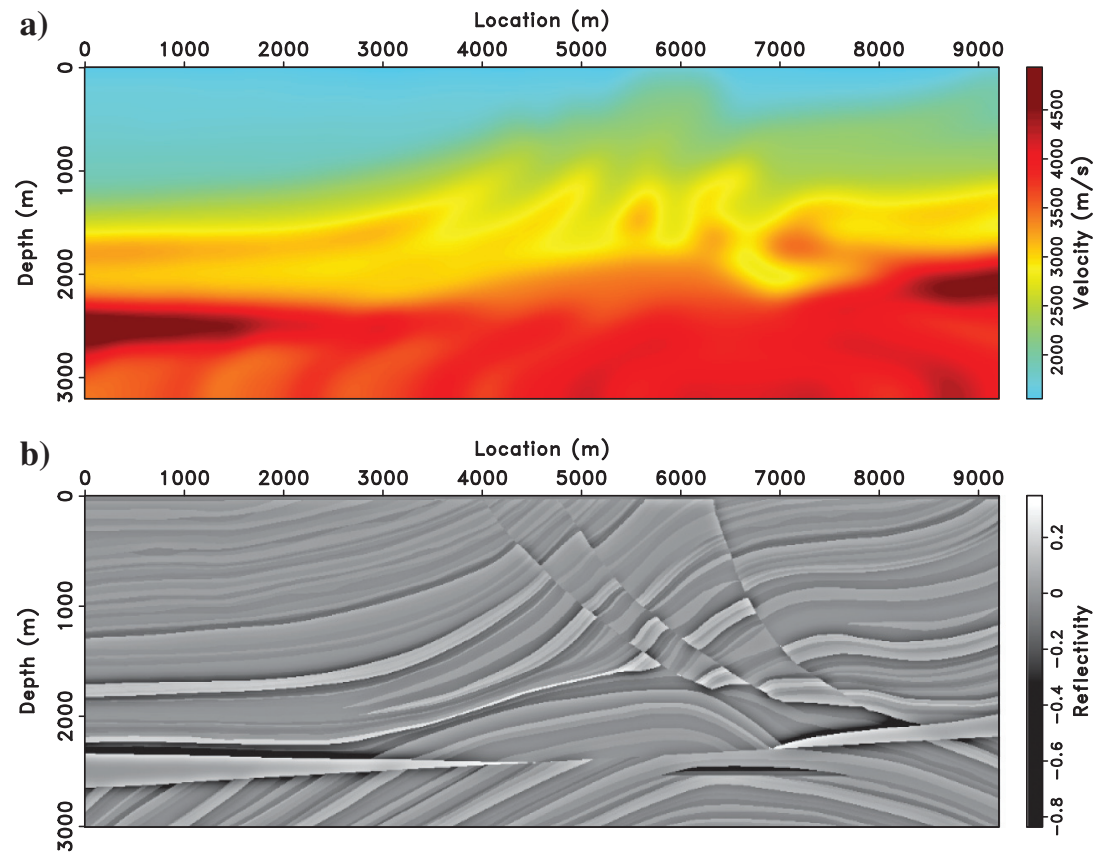

Figure 1. Marmousi model. (a) Background velocity model obtained by smoothing the original Marmousi model and (b) reflectivity model obtained by taking the difference between the original and the background models. 


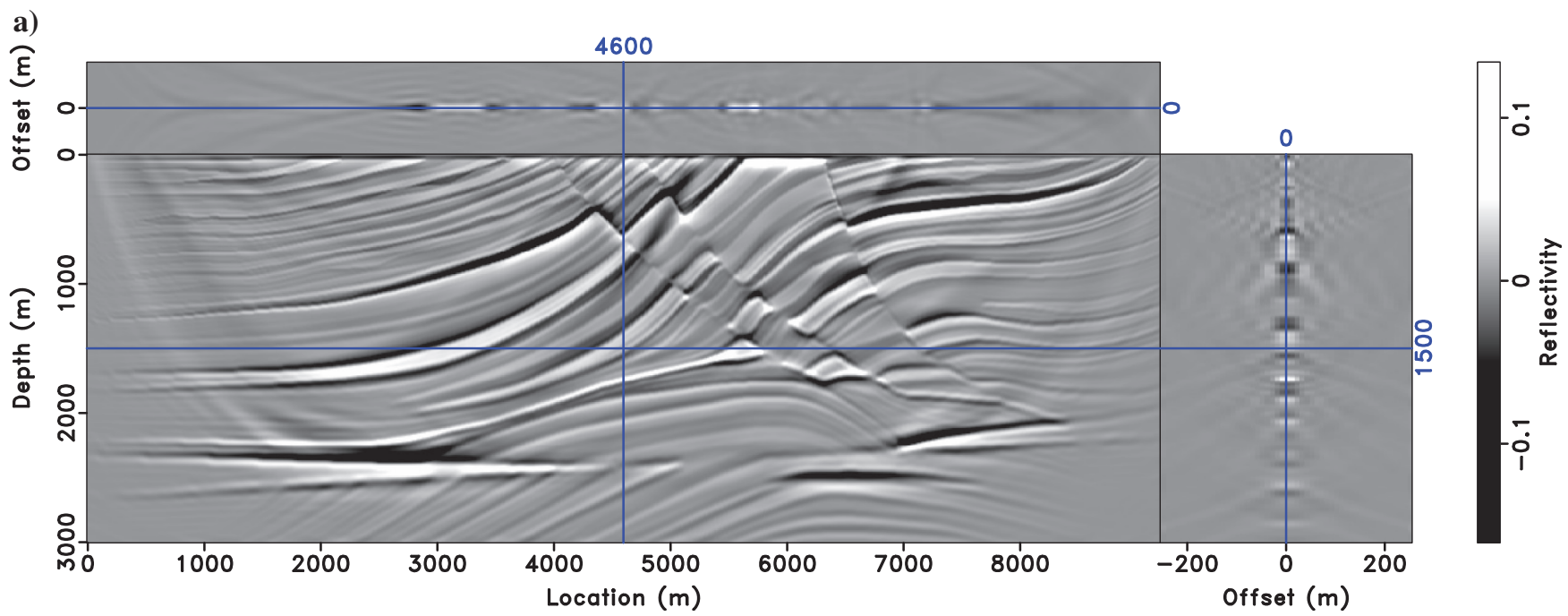

b)

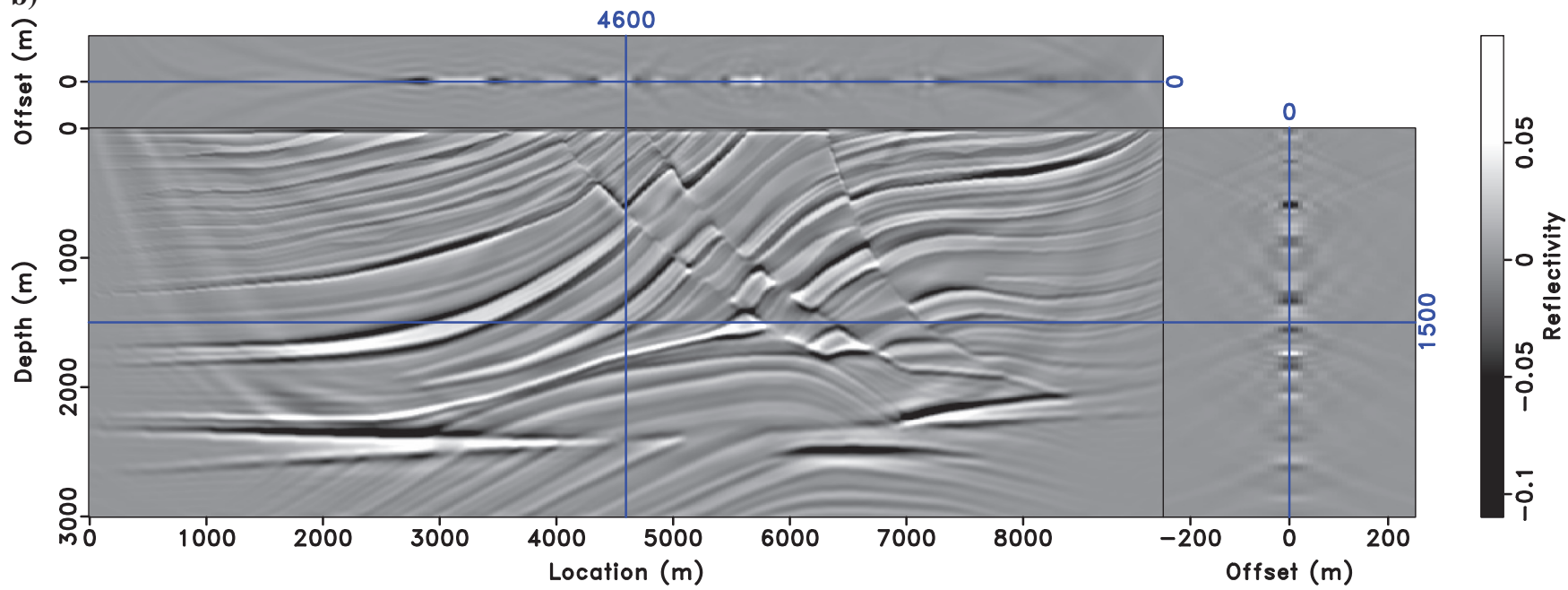

c)

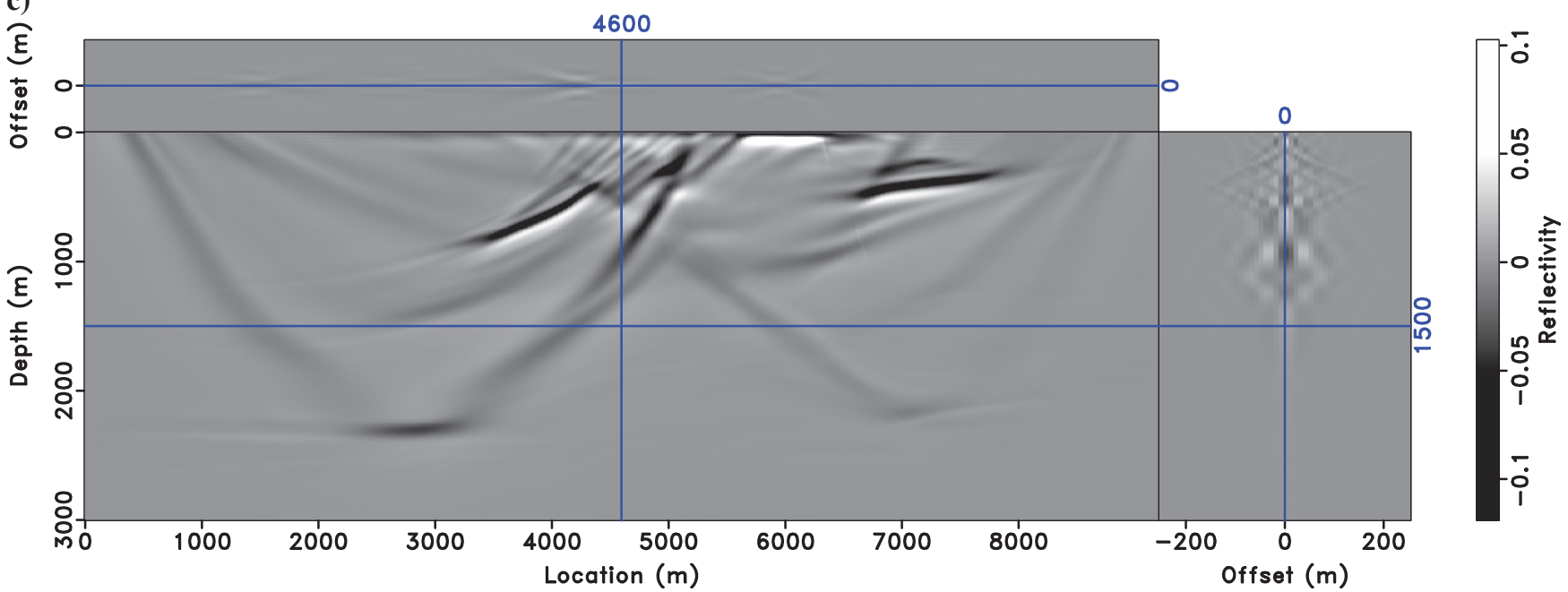

Figure 2. (a) The extended image inverted with formula 1, (b) the extended image inverted with formula 3 , and (c) the difference between the two extended images. 
erate the (Born) data with the same velocity model used to invert it via formulas 1 and 3, so the data to which the operators $P$ and $Q$ are applied are focused near $h=0$. Thus, these two operators act as approximate identities and are neglected in our computations.

Figure 1 shows the smoothed background velocity model and reflectivity model. The model is discretized on a $301 \times 921$ grid with a spacing of $10 \mathrm{~m}$ in the horizontal and vertical directions. The generated Born data have 231 common-shot gathers every $40 \mathrm{~m}$, and each shot has 921 receivers every $10 \mathrm{~m}$. The simulation uses a $2.5-5-30-35 \mathrm{~Hz}$ band-pass wavelet with $1 \mathrm{~ms}$ temporal sampling. The recording length is $4 \mathrm{~s}$.

Formulas 2 and 3 are applied on the Born data for the Marmousi model. The extended images have a subsurface offset range [-250 m: $250 \mathrm{~m}$ ] with $10 \mathrm{~m}$ spacing. Figure 2 plots the extended inversion produced by the two formulas and their difference. The comparison illustrates that both formulas for the approximate inversion express the same nature and produce very similar results. The difference mainly corresponds to the energy out of the asymptotic framework, e.g., the diving wave and refractions. As explained by Hou and Symes (2015b), the physical model can also be recovered by stacking along the subsurface offset axis (nonextended inversion). We plot the stacked images and their difference in Figure 3. These two stacked images are visually almost identical. The artifacts that appear in the extended images also cancel during the stacking process. The middle trace extracted from the reflectivity model, and the two stacked images (Figure 4a) further show the similarity of the two formulas. To further verify the effectiveness of the approximate inverse operators, we apply an extended Born modeling operator on the inverted extended images to resimulate the data. Figure 5 plots the middle shot of the original data, the resimulated data for the extended images using formulas 1 and 3, and the difference between the two resimulated data. The approximate inversion using both formulas produces almost the same level of relative data misfit compared with the original data. The middle trace (zero offset) extracted from the middle shot of the original data and two resimulated data confirms this view more clearly (Figure 4b). The substantial difference, especially at a large offset, between the two resimulated data (Figure 5d) results from the energy out of the asymptotic framework and shows the effect of the different $\Psi D O s$ in two formulas.

\section{DISCUSSION}

We thank Fons ten Kroode for pointing out that the asymptotic inverse 3 may be derived easily from equations 7 and 8 in ten Kroode (2014), which summarize an argument analogous to that explained here.
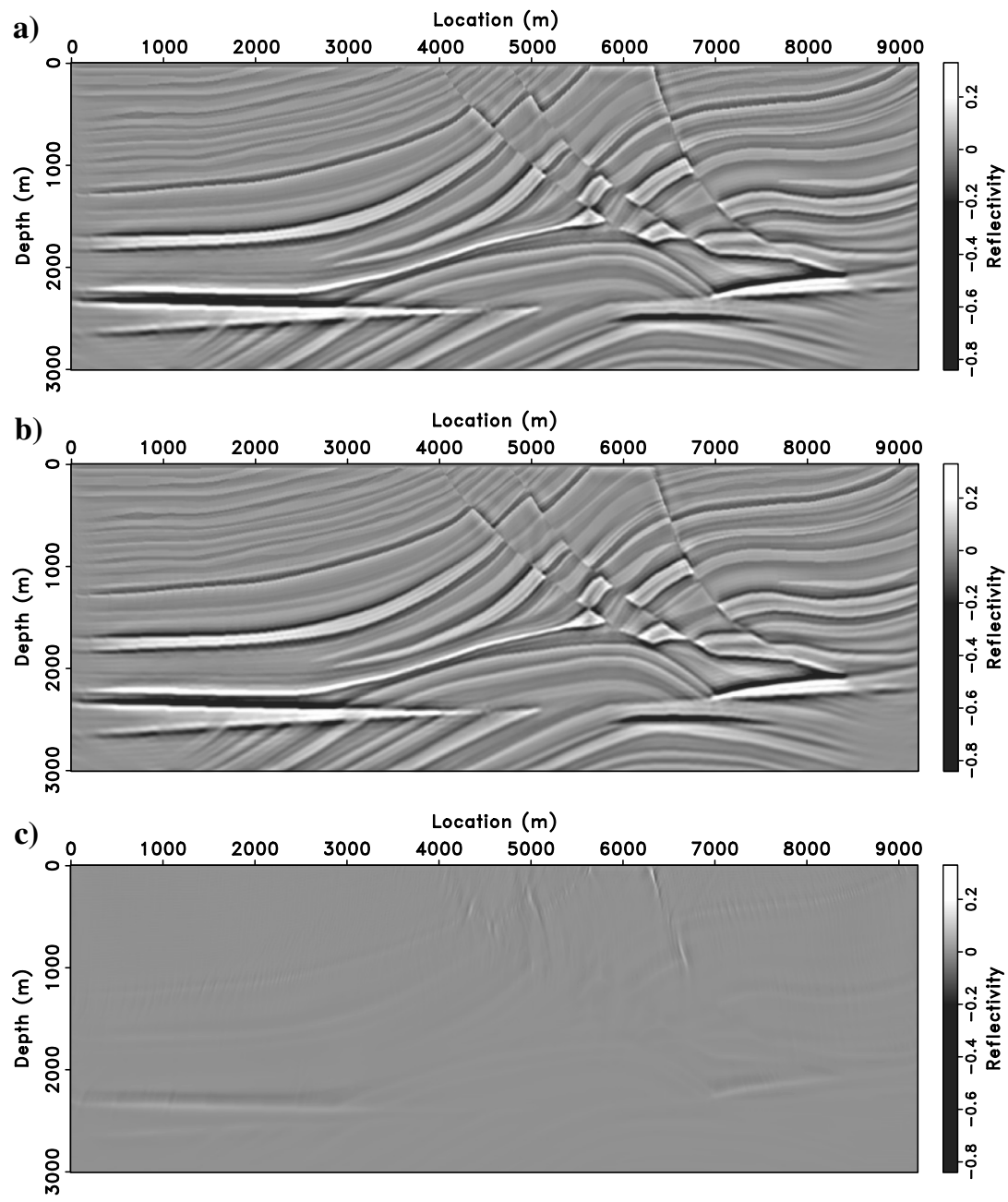

Figure 3. (a) Stacked image corresponding to the extended image in Figure 2a, (b) stacked image corresponding to the extended image in Figure $2 \mathrm{~b}$, and (c) the difference between ( $\mathrm{a}$ and $\mathrm{b}$ ). 
The operator defined by equation 1 is the adjoint of $\bar{F}$ in weighted norms on model and data spaces defined by $W_{\text {model }}$ and $W_{\text {data. }}$. Because the weighted normal operator $\bar{F}^{\dagger} \bar{F}$ is approximately the identity, the operator $\bar{F}$ is approximately unitary in the norms defined by the weight operator 2, so that Krylov subspace iterations such as
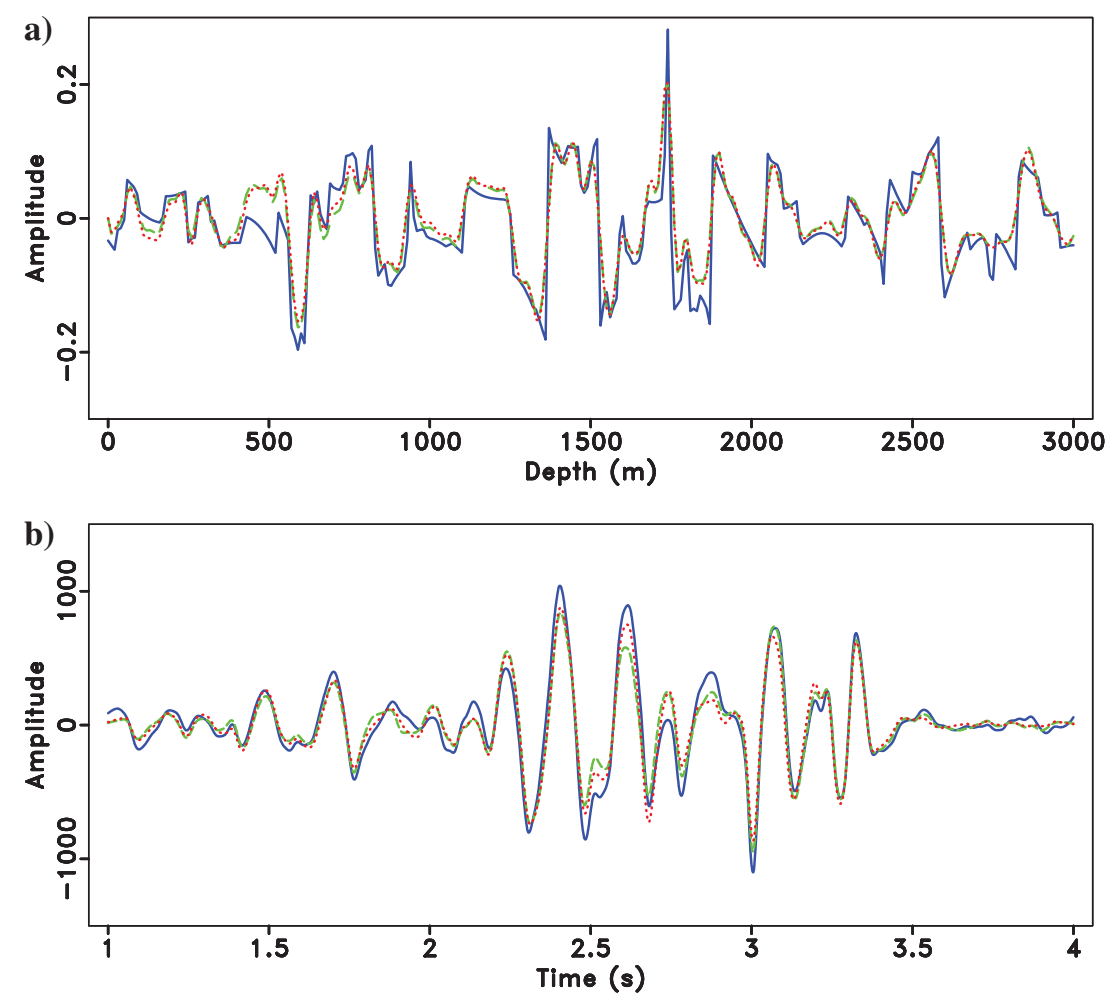

Figure 4. Middle trace comparison. (a) Model comparison: The blue line is the reflectivity model, the green line is the stacked image using formula 1, and the red line is the stacked image using formula 3. (b) Data comparison: The blue line is the original data, the green line is the resimulated data corresponding to the original formula, and the red line is the resimulated data corresponding to the new formula.
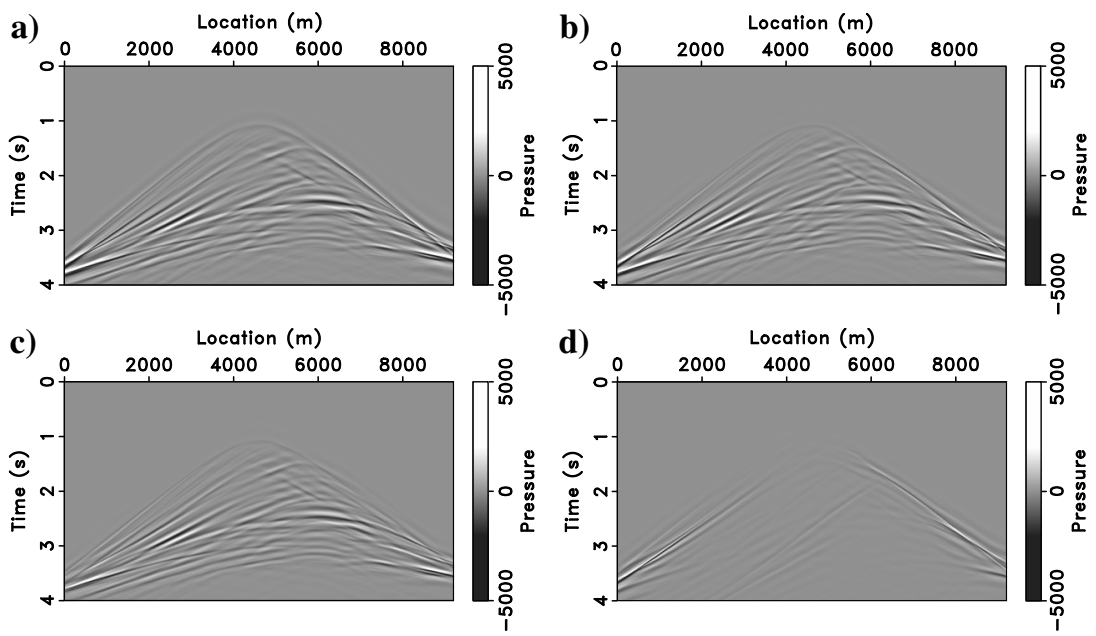

Figure 5. The middle shot of (a) original Born data, (b) resimulated Born data corresponding to the extended image in Figure $2 \mathrm{a}$, (c) resimulated Born data corresponding to the extended image in Figure $2 b$, and (d) the difference between (b and c). conjugate gradients converge very rapidly to solutions of the exverse operator shown in equation 3 can play a similar role in accelerating Krylov methods.

The $\Psi$ DOs $P$ and $Q$ are in principle necessary for asymptotically accurate recovery of unfocused extended models via 1 and 3. These operators have a low approximate rank, and efficient algorithms exist for approximating their action (Bao and Symes, 1996; Demanet and Ying, 2011). However, all of these algorithms have at least the complexity of the Fourier transform, so in fact they have a substantial cost, especially in $3 \mathrm{D}$. Moreover, only approximations may be computed; at present, the degree of approximation necessary to obtain an accurate approximate inverse is unknown. Experience so far suggests that for convergence acceleration of iterative algorithms, it may be possible to ignore $P$ as we have done here.

The derivations presented here are in principle valid only in the absence of triplications (multiple wave arrivals). However, there is ample evidence that this restriction is not really necessary (Stolk et al., 2009; ten Kroode, 2012, 2014). The absence of turning rays is a more fundamental limitation. To include them would require inclusion of vertical subsurface offsets (Biondi and Symes, 2004).

\section{CONCLUSION}

In this paper, we have derived a formula for an approximate extended Born inversion, whose additional cost beyond that of extended RTM is negligible. By theoretical derivation and numerical experiment, we have demonstrated that the operator derived here, while substantially less expensive to implement (especially in 3D), achieves the same order of asymptotic accuracy as that described in our previous work.

\section{ACKNOWLEDGMENTS}

We are grateful to the sponsors of The Rice Inversion Project for their long-term support, and to Shell International Exploration and Production Inc. for its support of J. Hou's Ph.D. research. We thank F. ten Kroode for inspiring our work. We thank J. Sheiman, H. Kuehl, and P. Shen for very helpful discussions. We have benefited greatly from the computational resources provided by the Texas Advanced Computing Center and the Rice University Research Computing Support Group. The Madagascar opensource software package has been critically useful in our work. We appreciate the constructive comments from F. ten Kroode and S. Gray. 


\section{APPENDIX A \\ PSEUDODIFFERENTIAL APPROXIMATION OF THE GRT}

We show that equation 5 gives a high-frequency asymptotic approximation of GRT 4, by modifying the argument explained in Appendix A of Hou and Symes (2015b). This argument begins with the assumption that $\partial \phi / \partial z>0$, where the input function $u \neq 0$ (in the application, $u$ will be the velocity perturbation $\delta \bar{v}$ ). Clearly, only those $\left(x^{\prime}, z^{\prime}, h^{\prime}\right)$ contribute to the evaluation of $M_{a} u(x, z, h)$ for which of $\phi\left(x_{r}, x_{s}, x, z, h\right)=\phi\left(x_{r}, x_{s}, x^{\prime}, z^{\prime}, h^{\prime}\right)$ for some $x_{s}, x_{r}$. Solve this equation for $z^{\prime}=Z\left(x_{r}, x_{s}, x, z, h, x^{\prime}, h^{\prime}\right)-$ given the assumption, the solution is at least locally unique. Note that as a distribution in $\left(x^{\prime}, z^{\prime}, h^{\prime}\right)$,

$$
\begin{aligned}
& \delta\left(\phi\left(x_{r}, x_{s}, x, z, h\right)-\phi\left(x_{r}, x_{s}, x^{\prime}, z^{\prime}, h^{\prime}\right)\right) \\
& =\left(\frac{\partial \phi}{\partial z^{\prime}}\left(x_{r}, x_{s}, x^{\prime}, z^{\prime}, h^{\prime}\right)\right)^{-1} \\
& \times \delta\left(z^{\prime}-Z\left(x_{r}, x_{s}, x, h, z, x^{\prime}, h^{\prime}\right)\right) .
\end{aligned}
$$

Substitute the identity A-1 in the definition 4 and introduce the Fourier transform of $u$ to obtain

$$
\begin{aligned}
M_{a} u(x, z, h)= & \frac{1}{8 \pi^{3}} \int d k_{x^{\prime}} d k_{z^{\prime}} d k_{h^{\prime}} d x_{r} d x_{s} d x^{\prime} d h^{\prime} \\
& \times a\left(x_{r}, x_{s}, x, z, h, x^{\prime}, Z\left(x_{r}, x_{s}, x, h, z, x^{\prime}, h^{\prime}\right), h^{\prime}\right) \\
& \times\left(\left|\frac{\partial \phi}{\partial z}\right|^{-1}\left(x_{r}, x_{s}, x^{\prime}, Z\left(x_{r}, x_{s}, x, h, z, x^{\prime}, h^{\prime}\right), h^{\prime}\right)\right. \\
& \left.\left.\times e^{i k_{z^{\prime}}\left(x^{\prime}{ }^{\prime} x_{x^{\prime}}\right.}+h^{\prime}{ }^{\frac{k}{h_{h^{\prime}}}} k_{z^{\prime}}+Z\left(x_{r}, x_{s}, x, h, z, x^{\prime}, h^{\prime}\right)\right)\right) \hat{u}\left(k_{x^{\prime}}, k_{z^{\prime}}, k_{h^{\prime}}\right) .
\end{aligned}
$$

Apply the stationary-phase principle to evaluate the right side of equation A-2 as in equation HS-A4. At the stationary point, $x=x^{\prime}, z=z^{\prime}, h=h^{\prime}$, and (dropping the primes on the frequency variables)

$$
\left(k_{x}, k_{z}\right)\left\|\nabla_{x, z} \phi,\left(k_{h}, k_{z}\right)\right\| \nabla_{h, z} \phi
$$

From relation A-3 it follows that the wave vector $\left(k_{x}, k_{z}, k_{h}\right)$ determines the ray connecting the scattering points $(x \pm h, z)$ to the $\left(x_{r}, z_{r}\right),\left(x_{s}, z_{s}\right)$, respectively; hence, the latter become functions of the co-vector $\left(x, z, h, k_{z}, k_{z}, k_{h}\right)$. In terms of these functions, to leading order in $k_{z}, M_{a} u(x, z, h)$

$\simeq \int d k_{x} d k_{z} d k_{h}\left[\frac{2 \pi}{k_{z}}\right]^{2}\left[\frac{1}{8 \pi^{3}}\right] a\left(x_{r}\left(x, z, h, k_{x} / k_{z}, k_{h} / k_{z}\right), x_{s}\left(x, z, h, k_{x} / k_{z}, k_{h} / k_{z}\right), x, z, h, x, z, h\right)$ $\times\left|\frac{\partial \phi}{\partial z}\right|^{-1}\left(x_{r}\left(x, z, h, k_{x} / k_{z}, k_{h} / k_{z}\right), x_{s}\left(x, z, h, k_{x} / k_{z}, k_{h} / k_{z}\right), x, z, h\right) e^{i\left(x k_{x}+z k_{z}+h k_{h}\right)} \widehat{\delta \bar{v}}\left(k_{x}, k_{z}, k_{h}\right)$ $\times\left|\operatorname{det} \operatorname{Hess}\left(x_{r}\left(x, z, h, k_{x} / k_{z}, k_{h} / k_{z}\right), x_{s}\left(x, z, h, k_{x} / k_{z}, k_{h} / k_{z}\right), x, z, h\right)\right|^{-1 / 2}$.

The Hessian factor $\mid$ det Hess $\left.\right|^{-1 / 2}$ is buried in HS-A27 and HS-A28: We fish it out as

$$
\mid \operatorname{det} \text { Hess }\left.\right|^{-1 / 2}=-\frac{v^{2}}{2}\left(\frac{\partial \alpha_{s}}{\partial x_{s}} \frac{\partial \alpha_{r}}{\partial x_{r}}\right)^{-1}\left(\frac{\partial \phi}{\partial z}\right)^{2} \frac{1}{Q}
$$

in which $\alpha_{s}$ and $\alpha_{r}$ are the ray takeoff angles at source and receiver positions; hence, they are also functions of $\left(x, z, h, k_{x}, k_{z}, k_{h}\right)$. Here, the arguments are suppressed temporarily. $Q=Q\left(x, z, h, k_{x}, k_{z}, k_{x}\right)$ is defined in terms of the $z$-components of ray slowness

$$
q_{r}=\frac{\partial T}{\partial z}\left(x_{r}, z_{r}, x+h, z\right), q_{s}=\frac{\partial T}{\partial z}\left(x_{s}, z_{s}, x-h, z\right)
$$

by the quantity in square brackets in HS-A28, multiplied by $(\partial \phi / \partial z)^{-2}$

$$
Q=\frac{v^{2}}{\left(q_{r}+q_{s}\right)^{2}}\left[\frac{q_{r}^{2}}{v_{-}^{2}}+\frac{q_{s}^{2}}{v_{+}^{2}}+\left(\frac{1}{v_{-}^{2}}+\frac{1}{v_{+}^{2}}\right) q_{s} q_{r}\right],
$$

in which $v_{ \pm}=v(x \pm h, z), v=v(x, z)$. The relation HS-A21 defines $q_{r}, q_{s}$ in terms of $v, v_{ \pm}$and $\partial \phi / \partial z$, and HS-A24, HS-A25, and HS-A26 define the $\partial \phi / \partial z$, as functions of $\left(x, z, h, k_{x}, k_{z}, k_{h}\right)$. The reader will note that $Q$ is dimensionless, and it is homogeneous of degree zero in $\left(k_{x}, k_{z}, k_{h}\right)$; hence, it is really a function of $\left(k_{x} / k_{z}, k_{h} / k_{z}\right)$. A bit of algebra using the quoted identities also establishes that

$$
Q\left(x, z, 0, k_{x} / k_{z}, k_{h} / k_{z}\right)=1 \text {. }
$$

Combining these results with equation A-4, we obtain equation 5 .

\section{APPENDIX B}

\section{AN ALTERNATIVE FORMULA FOR APPROXIMATE INVERSION}

Applying approximation 5 to equation 14, we obtain

$$
\begin{aligned}
& \left(I_{t} \bar{F}\right)^{*}\left(D_{t} I_{t} D_{z_{s}} I_{t} D_{z_{r}} I_{t} \bar{F}\right) \delta \bar{v}(x, z, h) \\
& \simeq \pi v(x, z)^{-4} \int d k_{x} d k_{z} d k_{h} e^{i\left(x k_{x}+z k_{z}+h k_{h}\right)} \widehat{\delta \bar{v}}\left(k_{x}, k_{z}, k_{h}\right) \\
& \quad \times\left[\left(\frac{\partial \alpha_{r}}{\partial x_{r}} \frac{\partial \alpha_{s}}{\partial x_{s}}\right)^{-1} a_{r}^{2} a_{s}^{2} \frac{\cos \theta_{r}}{v_{r}} \frac{\cos \theta_{s}}{v_{s}}\right] \\
& \quad \times\left(x_{r}\left(x, z, h, k_{x} / k_{z}, k_{h} / k_{z}\right), x_{s}\left(x, z, h, k_{x} / k_{z}, k_{h} / k_{z}\right), x, z, h\right) \\
& \quad \times \frac{i}{k_{z}} Q^{-1}\left(x, z, h, k_{x} / k_{z}, k_{h} / k_{z}\right) .
\end{aligned}
$$

To evaluate the right side of equation B-1, we use relations HSB6 and HS-B7

$$
a_{r}^{2} \frac{\cos \theta_{r}}{v_{r}}\left(\frac{\partial \alpha_{r}}{\partial x_{r}}\right)^{-1}=\frac{1}{8 \pi^{2}}=a_{s}^{2} \frac{\cos \theta_{s}}{v_{s}}\left(\frac{\partial \alpha_{s}}{\partial x_{s}}\right)^{-1}
$$

Substitution of these identities in B-1 leads directly to equation 16 , hence leading to the asymptotic inverse formula 3 as described in the "Theory" section. 
SB

How and Cymes

REFERENCES

Bap, G., and W. Symes, 1996, Computation of pseudo-differential operators: SIAM Journal on Scientific Computation, 17, 416-429, do: 10.1137/ S1064827593258279.

Beylkin, G., 1985, Imaging of discontinuities in the inverse scattering problem by inversion of a causal generalized Radon transform: Journal of Mathematical Physics, 26, 99-108, doe: 10.1063/1.526755.

Biondi, B., and W. Symes, 2004, Angle-domain common-image gathers for migration velocity analysis by wavefield-continuation imaging: Geophysiss, 69, 1283-1298, doe: 10.1190/1.1801945.

Bleistein, N., 1987, On the imaging of reflectors in the earth: Geophysics, 52, 931-942, do: 10.1190/1.1442363.

Bourgeois, A., M. Bourget, P. Lailly, M. Poult, P. Ricarte, and R. Versteeg, 1991, Marmousi, model and data, in R. Versteeg and G. Grau, eds., The Marmousi experience: Proceeding of the 1990 EAEG workshop on proctical aspects of seismic data inversion, 5-16.

Demanet, L., and L. Yong, 2011, Discrete symbol calculus: SIAM Review, 53, 71-104, doe: 10.1137/080731311.

Fou, J., and W. Symes, 2014, An approximate inverse to the extended Born modeling operator: 84th Annual International Meeting, SEG, Expanded Abstracts, 3784-3789.

Fou, J., and W. Symes, 2015a, Accelerating extended least squares migraion with weighted conjugate gradient iteration: 85th Annual International Meeting, SEG, Expanded Abstracts, 4243-4248.

How, J., and W. Symes, 2015b, An approximate inverse to the extended Born modeling operator: Geophysics, 80, no. 6, R331-R349, do: 10.1190/ geo2014-0592.1.
Hoo, J., and W. Symes, 2016a, Accelerating extended least-squares migraion with weighted conjugate gradient iteration: Geophysics, 81, no. 4, S165-S179, do: 10.1190/geo2015-0499.1.

Hoo, J., and W. Symes, 2016b, Accelerating least squares migration with weighted conjugate gradient iteration: 78th Annual International Conference and Exhibition, EAGE, Extended Abstracts, P104, oi: 10.3997/ 2214-4609.201601375.

Operto, M. S., S. Xu, and G. Lambaré, 2000, Can we quantitatively image complex structures with rays?: Geophysics, $\mathbf{6 5}, 1223-1238$, dor: 10.1190/ 1.1444814 .

Sava, P., and S. Fomel, 2003, Angle-domain common-image gathers by wavefield continuation methods: Geophysics, 68, 1065-1074, doa: 10 $.1190 / 1.1581078$.

Stork, C. C., M. V. de Hoop, and W. Symes, 2009, Kinematics of shot-geophone migration: Geophysics, 74, no. 6, WCA18-WCA34, do: 10.1190/ 1.3256285 .

Taylor, M., 1981, Pseudo differential operators: Princeton University Press. ten Kroode, F., 2012, A wave-equation-based Kirchhoff operator: Inverse Problems, 28, 115013.

ten Kroode, F., 2014, A Lie group associated to seismic velocity estimation: Proceedings of the Inverse Problems: From Theory to Application, Intitote of Physics.

Xu, S., Y. Chang, and B. Tang, 2011, 3D angle gathers from reverse time migration: Geophysics, 76, no. 2, S77-S92, doit: 10.1190/1.3536527.

Chang, Y., J. Sun, and S. H. Gray, 2007, Reverse time migration: Amplitude and implementation issues: 77 th Annual International Meeting, SEG, Exbanded Abstracts, 2145-2149. 